\title{
ISOTERMAS DE SORÇÃO DE SEMENTES DE Eucaliptus grandis E Pinus taeda ${ }^{1}$
}

\author{
JUSSARABERTHO FANTINATTI ${ }^{2}$, ROBERTO USBERT ${ }^{3}$, FERNANDO PEDRO REISBROD ${ }^{4}$
}

\begin{abstract}
RESUMO - As sementes florestais devem ser armazenadas adequadamente, a fim de reduzir ao mínimo o processo de deterioração. É possível estabelecer uma relação entre o teor de água livre na semente e sua conservação, expresso pela atividade de água, através da relação entre a pressão de vapor de água em equilíbrio sobre a semente e a pressão de vapor de água pura, à mesma temperatura. Uma isoterma é uma curva que descreve, em uma umidade específica, a relação de equilíbrio de uma quantidade de água sorvida por componentes da semente e a pressão de vapor ou umidade relativa, a uma temperatura específica. O objetivo deste trabalho foi estudar as isotermas de sorção para sementes de Eucalyptus grandis e Pinus taeda, que são espécies florestais de interesse econômico. Os graus de umidade das sementes foram ajustados, antes do armazenamento, usandose dessecadores com sílica gel ou através de reidratação sobre água. Para o controle da quantidade de água removida ou absorvida, as subamostras foram pesadas periodicamente, sendo o processo encerrado ao ser atingido o peso correspondente ao grau de umidade final desejado para cada tratamento. Foram realizadas determinações de umidade e de atividade de água e utilizados diferentes modelos de equações empíricas que correlacionam dados experimentais das isotermas de sorção em materiais biológicos. O melhor ajuste das isotermas de sorção foi alcançado pelos modelos de três parâmetros de Langmuir e de quatro parâmetros de Peleg para sementes de E. grandis e de $P$. taeda, respectivamente.
\end{abstract}

Termos para indexação: espécies florestais, atividade de água, conservação, grau de umidade.

\section{SORPTION ISOTHERMS FOR Eucaliptus grandis AND Pinus taeda SEEDS}

\begin{abstract}
Forest seeds must be adequately stored in order to minimise the process of deterioration. The level that limits the water in stored seeds is called the moisture equilibrium. It is possible to establish a relation between seed free water content and conservation, expressed by the water activity,, given by the relation between seed water and pure water vapour pressures, at the same temperature. An isotherm is a curve that describes, in specific moisture, the equilibrium of an amount of water sorbed by seed components and the vapour pressure or relative humidity, at a specific temperature. The main aim of this study was to analyse sorption isotherms for Eucalyptus grandis and Pinus taeda, which are forest species of economic interest. Seed moisture contents were adjusted before storage, using silica gel or rehydration above water. Seed subsample weights were recorded periodically to control the amounts of removed or sorbed water. The process was over when the corresponding weight to the desired seed moisture content had been achieved. Seed moisture content and water activity determinations have been carried out and different
\end{abstract}

\footnotetext{
${ }^{1}$ Submetido em 06/04/2004. Aceito para publicação em 02/06/2005. Projeto financiado pela FAPESP

${ }^{2}$ Bióloga, Doutora em Engenharia Agrícola, UNICAMP/FEAGRI/Tecnologia Pós-colheita, Cx.Postal 6011, 13083-970, Campinas-SP. jbfant@ig.com.br
}

\footnotetext{
${ }^{3}$ Eng $^{\circ}$ Agr $^{\circ}$, PhD., CDA/SAA; Professor Colaborador da UNICAMP/FEAGRI/ Tecnologia Pós-Colheita., Campinas, SP. usberti@cati.sp.gov.br

${ }^{4}$ Eng. Agrícola, Dr., UNICAMP/FEAGRI, Campinas/SP. brod@agr.unicamp.br
} 
empirical equation models were used aiming to correlate experimental data of sorption isotherms of the seed samples. The best adjustment of the sorption isotherms was achieved through the Langmuir 3-parameter model and the Peleg 4-parameter model for seeds of E. grandis and P. taeda, respectively.

Index terms: forest species, water activity, conservation, moisture content.

\section{INTRODUÇÃO}

O Brasil ocupa posição de destaque no cenário tecnológico da silvicultura internacional, principalmente em relação às espécies Eucalyptus spp. e Pinus spp., apresentando uma grande concentração de empresas de produção de papel e celulose e de confecção de móveis, sendo grande a preocupação com a conservação dessas espécies para o reflorestamento.

Esta atividade do agronegócio pode proporcionar bom retorno econômico devido à elevada produtividade, ao alto valor econômico dos produtos, à disponibilidade de terras e de mão-de-obra, apesar da redução dos reflorestamentos em alguns estados brasileiros, ocorridos na década passada.

Para se reduzir ao mínimo o processo de deterioração, as sementes devem ser armazenadas adequadamente, garantindo assim a manutenção do estoque regulador de sementes para os anos subsequentes de baixa produção.

Como todo material higroscópico, as sementes cedem ou absorvem água do ar que as envolve; assim, se a pressão de vapor de água na semente for menor do que a do ar, ocorre a absorção de umidade (sorção) e, no caso inverso, a semente cede água para o ar (dessorção). Quando a pressão de vapor de água da superfície da semente se iguala à pressão do ar ambiente, obtém-se a umidade de equilíbrio (Nellist e Hugues, 1973).

Por serem altamente higroscópicas, as sementes têm comportamento diferenciado nas isotermas de sorção. Existem muitas equações empíricas que correlacionam dados experimentais das isotermas de sorção de materiais biológicos, através de modelos matemáticos.

Sementes ricas em óleo apresentam graus de umidade de equilíbrio mais baixos em relação às sementes amiláceas, quando armazenadas em condições ambientais semelhantes, pois absorvem menos água, por serem hidrófobas (Brooker et al., 1992). Resultados semelhantes foram obtidos por Benedetti e Jorge (1987), para sementes de amendoim (alto teor de lipídios), que apresentaram menor umidade de equilíbrio quando comparadas com as sementes de arroz, milho, soja e trigo, a uma mesma temperatura.
Chung e Pfost (1967) verificaram que, para sementes de milho, entre os graus de umidade de 4 e $20 \%$ a $31^{\circ} \mathrm{C}$, o calor de sorção/desorção estaria entre 10,5 e 16kcal.mol ${ }^{-1}$.

A temperatura tem efeito significativo no conteúdo de umidade de equilíbrio. Em milho, a umidade de equilíbrio a $70 \%$ UR (umidade relativa do ar) é $15,6 \%$, a $4,4^{\circ} \mathrm{C}$ e $10,3 \%$, a $60^{\circ} \mathrm{C}$. Outras sementes apresentam similaridade no comportamento, sendo que um aumento na temperatura a determinada UR constante, diminui o conteúdo de umidade de equilíbrio (Brooker et al., 1992).

O objetivo deste trabalho foi estudar as isotermas de sorção para sementes das espécies florestais Eucalyptus grandis A.W.Hill ex Mainden e Pinus taeda L.

\section{MATERIAL E MÉTODOS}

O presente estudo foi realizado no Laboratório de Tecnologia Pós-Colheita da Faculdade de Engenharia Agrícola, Universidade Estadual de Campinas, SP, no período de março de 2000 a dezembro de 2003. As sementes foram adquiridas no Instituto de Pesquisas e Estudos Florestais, IPEF, Piracicaba, São Paulo.

Os graus de umidade das sementes foram ajustados, a partir de seu valor inicial, em oito níveis antes do armazenamento, usando-se dessecadores com sílica gel, constantemente regenerada ou através de umidificação sobre água (camada de água de $4 \mathrm{~cm}$ em sua parte inferior), ambos a $25^{\circ} \mathrm{C}$. Para o controle da quantidade de água removida ou sorvida durante a secagem ou reidratação das sementes, as subamostras foram pesadas periodicamente. O processo foi encerrado ao ser atingido o peso correspondente ao grau de umidade final desejado para cada tratamento.

A equação utilizada para se obter os valores desejados foi a adotada por Valentini (1992): $\mathrm{Mf}=((\mathrm{PBi}-\mathrm{T}) * \mathrm{Mi}+$ $100 *(\mathrm{PBf}-\mathrm{PBi})) /(\mathrm{PBf}-\mathrm{T})$ onde, $\mathrm{Mf}=$ grau de umidade final (\%, b.u.); $\mathrm{PBi}=$ massa bruta inicial em gramas; $\mathrm{T}=$ tara do saco de filó (Pinus) e placa de petri (Eucalyptus); $\mathrm{Mi}=$ grau de umidade inicial (\%, b.u.); PBf = massa bruta final em gramas. Após um período de quatro dias para homogeneização, o grau de umidade das sementes foi determinado; utilizando- 
se o método da estufa com circulação de ar a $105 \pm 3^{\circ} \mathrm{C}$ por 24 horas e a $130 \pm 0,3^{\circ} \mathrm{C}$ por uma hora (sementes moídas), com duas subamostras de $5 \mathrm{~g}$ por repetição, conforme Brasil (1992).

A determinação da atividade de água (Aw) foi realizada no Centro de Tecnologia de Embalagem do Instituto de Tecnologia de Alimentos, utilizando-se um higrômetro baseado em psicrometria, de marca Decagon-Aqualab, com resolução de 0,01Aw. Este equipamento é acoplado a um banho termostatizado, de marca Brookfield, modelo TC 500, com resolução de $0,1^{\circ} \mathrm{C}$. As determinações foram feitas a $25 \pm$ $0,3^{\circ} \mathrm{C}$, usando-se três subamostras de sementes moídas para cada grau de umidade. O princípio de medição do aparelho é a determinação da temperatura do ponto de orvalho (Aqualab, 1997).

Equações empíricas foram usadas para correlacionar as isotermas de sorção das sementes, a saber: Modelo de Peleg: Modelo empírico de quatro parâmetros que visa conjugar duas tendências em uma equação (Peleg, 1993): $\mathrm{X}_{\mathrm{eq}}=\mathrm{k}_{1} \cdot \mathrm{a}_{\mathrm{w}}{ }^{\mathrm{n}_{1}}+\mathrm{k}_{2} \cdot \mathrm{a}_{\mathrm{w}}{ }^{\mathrm{n}_{2}}$ onde, $\mathrm{X}_{\mathrm{eq}}=$ teor de água de equilíbrio, kg.kg-1; $\mathrm{Aw}=$ atividade de água, adimensional; $\mathrm{k}_{1}$, $\mathrm{k}_{2}, \mathrm{n}_{1}$ e $\mathrm{n}_{2}=$ constantes. A restrição para esta equação é que $\mathrm{n}_{1}$ $<1$ e $\mathrm{n}_{2}>1$; Modelo de Bet (Brunauer, Emmet e Teller): Analisando aspectos da natureza química da umidade, Langmuir, Brunauer, Emmet e Teller (Bet) propõem, para camadas polimoleculares:

$$
X_{e q}=\frac{\left(X m \cdot C \cdot a_{w}\right) \cdot\left(1-(n+1) \cdot a_{w}{ }^{n}+n \cdot a_{w}{ }^{n+1}\right)}{\left(1-a_{w}\right) \cdot\left(1+(C-1) \cdot a_{w}-C \cdot a_{w}{ }^{n+1}\right)} \text { onde, } X_{e q}=\text { teor de }
$$

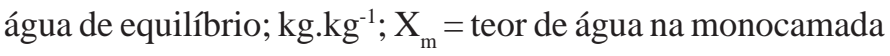
molecular, kg.kg ${ }^{-1} ; \mathrm{Aw}=$ atividade de água, adimensional; C, $\mathrm{n}$ = constantes (Park e Nogueira, 1992); Modelo de GAB (Gugghenheim, Anderson e de Boer): equação triparamétrica, que permite um melhor ajuste dos dados de sorção das sementes até a atividade de água de 0,9. A equação de GAB é descrita, segundo Van Der Berg (1984), como:

$\mathrm{X}_{\mathrm{eq}}=\frac{\mathrm{Xm} \cdot \mathrm{C} \cdot \mathrm{K} \cdot \mathrm{a}_{\mathrm{w}}}{\left(1-\mathrm{K} \cdot \mathrm{a}_{\mathrm{w}}\right) \cdot\left(1-\mathrm{K} \cdot \mathrm{a}_{\mathrm{w}}+\mathrm{C} \cdot \mathrm{K} \cdot \mathrm{a}_{\mathrm{w}}\right)}$ onde, $\mathrm{X}_{\mathrm{eq}}=$ teor de

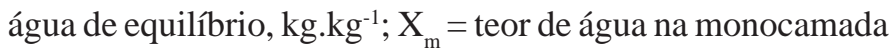

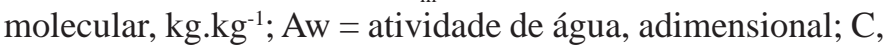
$\mathrm{K}$ = constantes de adsorção; Modelo de Langmuir: Langmuir (1918) considerou as condições de equilíbrio aplicada à água livre, propondo a equação: $X_{e q}=\frac{X M \cdot C \cdot a_{w}}{1+C \cdot a_{w}}$ onde, Aw
= atividade de água, adimensional; $\mathrm{X}_{\mathrm{eq}}=$ teor de água de

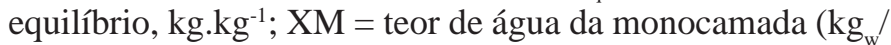
kg $_{s}$ ); C = constantes de adsorção; Modelo de Halsey: Helsey (1985) desenvolveu um modelo para a condensação $\mathrm{a}_{\mathrm{w}}=\exp \left(\frac{-\mathrm{A}}{\mathrm{X}_{\text {eq }} \mathrm{B}}\right)$ das camadas a uma distância relativamente grande da superfície: onde, $\mathrm{X}_{\mathrm{eq}}=$ teor de água

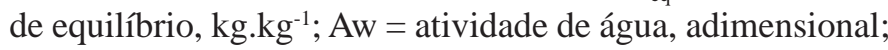
A, B = constantes. Modelo de Oswin: baseia-se na expansão matemática para curvas de formato sigmoidal, sendo um modelo empírico, definido por Chinnan e Beauchat (1985)

como: $\mathrm{X}_{\text {eq }}=\mathrm{A} \cdot\left(\frac{\mathrm{a}_{\mathrm{w}}}{1-\mathrm{a}_{\mathrm{w}}}\right)^{\mathrm{B}}$ onde, $\mathrm{X}_{\mathrm{eq}}=$ teor de água de equilíbrio, $\mathrm{kg}_{\mathrm{kg}} \mathrm{kg}^{-1} \mathrm{Aw}=$ atividade de água, adimensional; A, $\mathrm{B}=$ constantes.

\section{RESULTADOS E DISCUSSÃO}

As sementes de E. grandis perderam água mais rapidamente do que as de $P$. taeda no início da secagem (Figura 1), mostrando que com a velocidade mais lenta do processo, o grau de umidade das sementes apresentou relação inversa com o tempo de secagem. Hall (1980) afirmou que no processo ocorre aumento da temperatura, devido à transferência de calor não ser compensada pela transferência de massa de água no interior da semente; quando a taxa de transporte interno da água é menor do que a taxa de evaporação, esta velocidade é decrescente.

Após dois dias de secagem, as sementes de E. grandis apresentaram grau de umidade de $6,77 \%$, enquanto as de $P$. taeda atingiram 7,37\% (Figura 1). Provavelmente, esse fato pode ser atribuído às diferenças nos teores de lipídeos detectados para as espécies (24,2 e 52,3\%, respectivamente).

Para as duas espécies florestais, a secagem em sílica gel respondeu positivamente, sendo que as umidades mais baixas foram obtidas sem causar danos às sementes, principalmente para E. grandis, material de composição heterogênea que não suporta secagem em altas temperaturas. A eficiência deste método já foi comprovado em outras espécies vegetais por Xiaorong et al. (1998).

Para E. grandis e P. taeda, foram alcançados graus de umidade na faixa de 1 a 19\%, com umidade relativa de equilíbrio variando de 8 a 95\%. A atividade de água detectada 


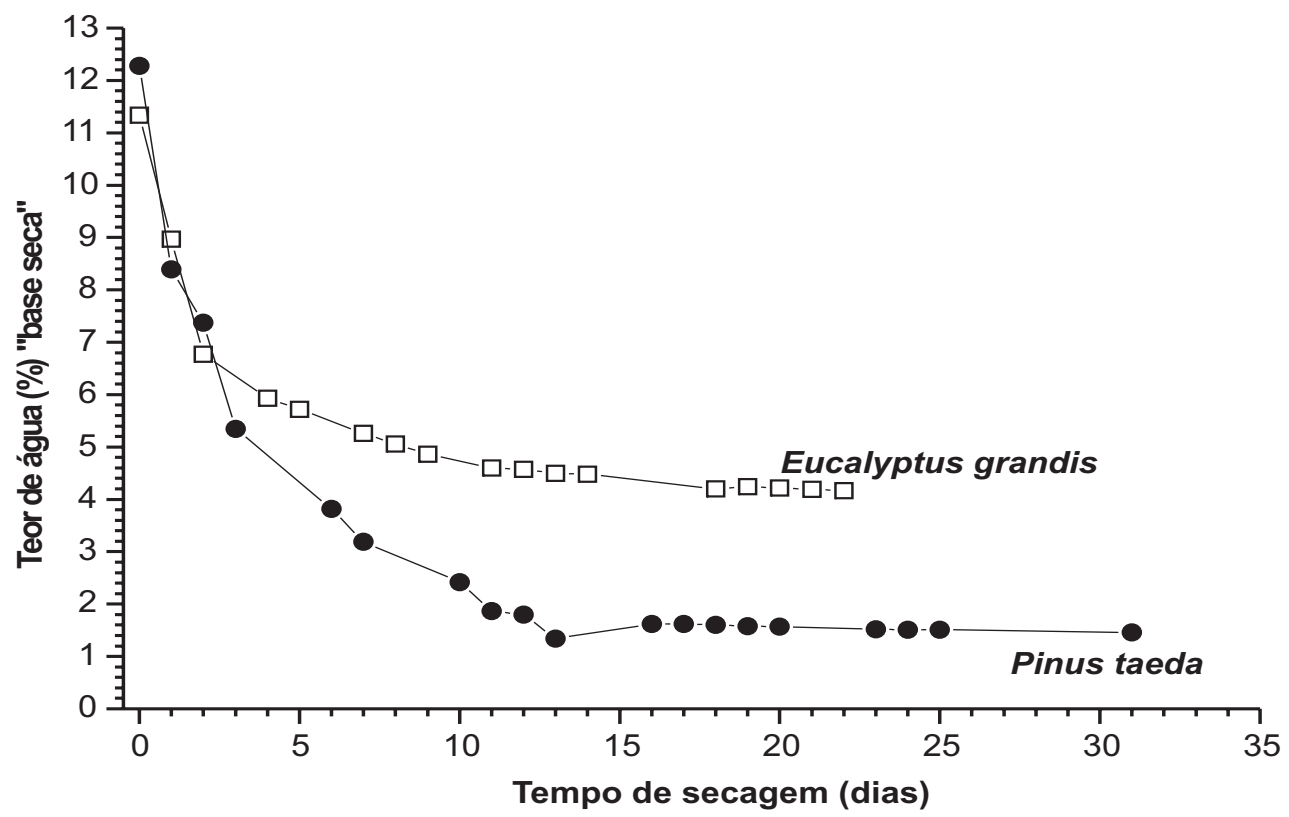

FIGURA 1. Grau de umidade em função do tempo de secagem de sementes de Eucalyptus grandis e Pinus taeda, em sílica gel, a $25^{\circ} \mathrm{C}$.

revela que as sementes de $P$. taeda são mais oleaginosas do que as de E. grandis. O conhecimento de isotermas de umidade de equilíbrio higroscópio das sementes é essencial por estarem diretamente ligadas ao armazenamento, secagem e comercialização (Roa e RossiI, 1977). As umidades relativas de equilíbrio detectadas para as duas espécies são apresentadas na Tabela 1.

A determinação e os módulos dos erros relativos médios foram testados nos modelos de Bet (Brunauer, Emmet e Teller); Bet Linear; Gab (Gugghenheim, Anderson e de Boer); Halsey modificado; Oswin, Langmuir e Peleg.

O melhor ajuste das isotermas foi alcançado pelo modelo de três parâmetros de Langmuir para E. grandis (erro relativo = $12,22 \%$ ), enquanto que para P. taeda, o modelo mais adequado foi o de quatro parâmetros de Peleg (erro relativo = 6,71\%). Esses resultados são apresentados na Tabela 2.

Usando os modelos de Langmuir e Peleg, foram obtidas isotermas de sorção e dessorção (Figuras 2 e 3) entre os valores experimentais e os previstos para as espécies, dependendo se os valores obtidos foram abaixo ou acima dos graus de umidade iniciais (11,34\%, para E. grandis e 12,28\%, para P. taeda).

As isotermas abrangem três regiões, que correspondem aos diferentes tipos de água ligada que ocorrem nos tecidos; a baixo grau de umidade, a água nas sementes é mantida por ligações muito fortes enquanto que, para umidades intermediárias e altas, a água é mantida por ligações fracas e
TABELA1. Valores de atividade de água (Aw) e graus de umidade de sementes, obtidos a $105 \mathrm{e} 130^{\circ} \mathrm{C}$, para $E$. grandis e P. taeda.

\begin{tabular}{|c|c|c|c|}
\hline \multirow{3}{*}{ Espécies } & \multicolumn{3}{|c|}{ Graus de umidade e Aw (\%) } \\
\hline & \multicolumn{2}{|c|}{ Temperaturas $\left({ }^{\circ} \mathrm{C}\right)$} & \multirow{2}{*}{ Aw } \\
\hline & 105 & 130 & \\
\hline \multirow{8}{*}{ Eucalyptus grandis } & 1,24 & 1,66 & 0,087 \\
\hline & 2,95 & 3,35 & 0,096 \\
\hline & 4,67 & 4,93 & 0,228 \\
\hline & 7,51 & 7,97 & 0,397 \\
\hline & 11,34 & 11,39 & 0,654 \\
\hline & 14,44 & 14,81 & 0,806 \\
\hline & 16,34 & 15,94 & 0,858 \\
\hline & 18,12 & 18,53 & 0,936 \\
\hline \multirow{8}{*}{ Pinus taeda } & 1,46 & 1,76 & 0,103 \\
\hline & 3,95 & 4,03 & 0,210 \\
\hline & 5,70 & 5,96 & 0,293 \\
\hline & 8,02 & 8,35 & 0,456 \\
\hline & 12,28 & 12,66 & 0,735 \\
\hline & 13,45 & 13,85 & 0,816 \\
\hline & 17,03 & 17,27 & 0,915 \\
\hline & 19,45 & 19,81 & 0,956 \\
\hline
\end{tabular}

a água multimolecular (Vertuccie Leopold, 1987).

As sementes de $P$. taeda apresentaram graus de umidade de equilíbrio mais baixos do que as de E. grandis. Sementes ricas em óleo apresentam graus de umidade de equilíbrio mais baixos em relação às sementes amiláceas. 
TABELA 2. Erro relativo médio nos modelos de isotermas de sorção para E. grandis e P. taeda.

\begin{tabular}{lcc}
\hline \multirow{2}{*}{ Modelo } & \multicolumn{2}{c}{ Erro (\%) } \\
\cline { 2 - 3 } & Eucalyptus & Pinus \\
\hline BET & 15,19 & 14,76 \\
BET LINEAR & 46,64 & 49,89 \\
GAB & 12,45 & 10,80 \\
HALSEY modificad & 51,01 & 40,31 \\
LANGMUIR* & $12,22^{*}$ & 9,27 \\
OSWIN & 35,98 & 29,19 \\
PELEG* $^{*}$ & 12,62 & $6,71^{*}$ \\
\hline
\end{tabular}

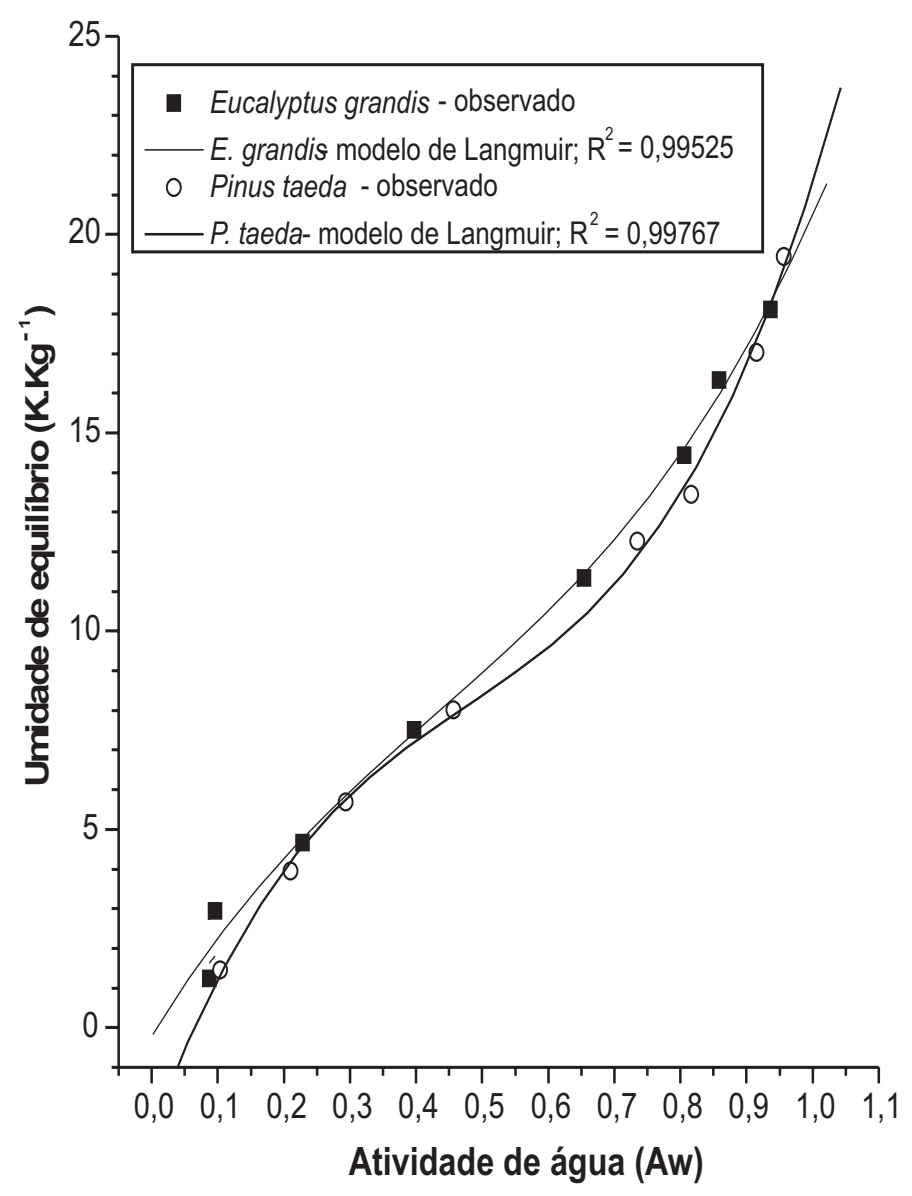

FIGURA2. Modelo de Langmuir de isotermas de sorção ajustado, aos valores experimentais para as sementes de Eucalyptus grandis e Pinus taeda.

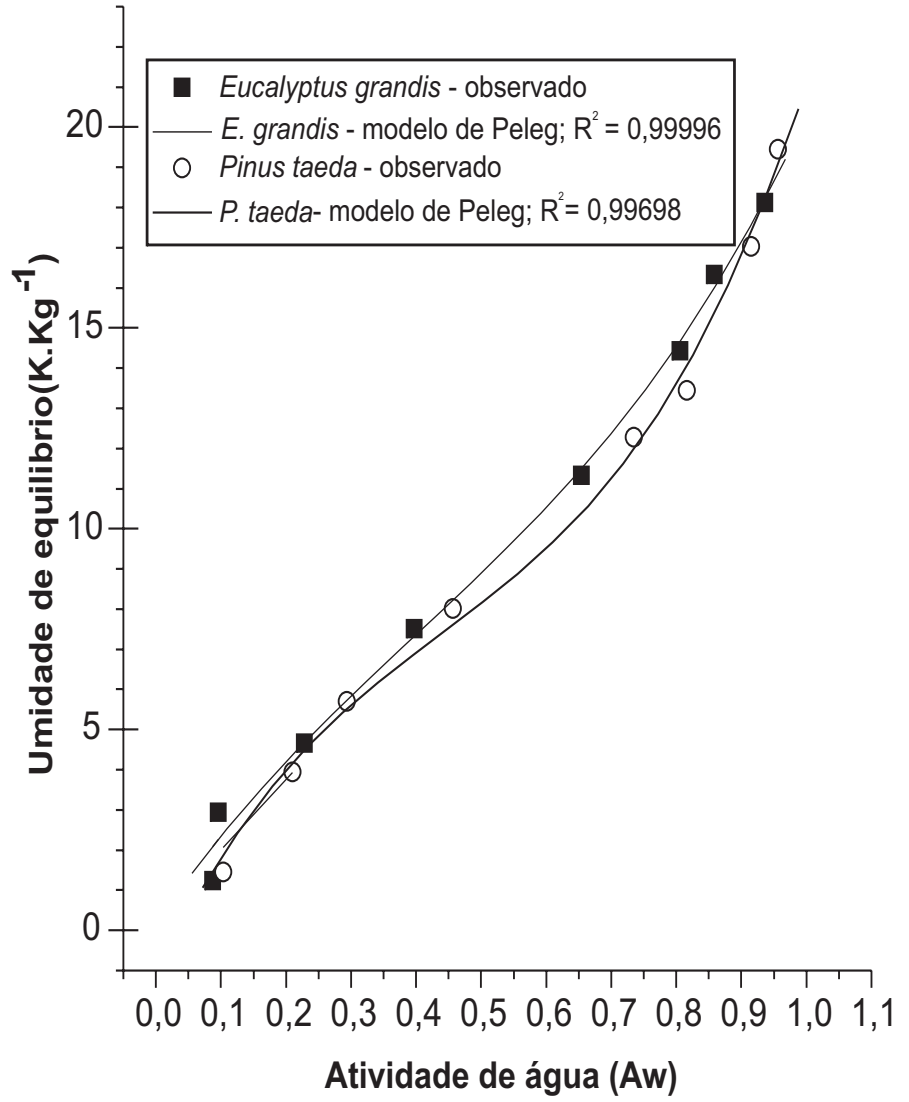

FIGURA 3. Modelo de Peleg de isotermas de sorção ajustado aos valores experimentais para as sementes de Eucalyptus grandis e Pinus taeda.

\section{CONCLUSÃO}

O melhor ajuste das isotermas de sorção foi alcançado através do modelo de três parâmetros de Langmuir para sementes de Eucalyptus grandis e do modelo de quatro parâmetros de Peleg para sementes de Pinus taeda.

\section{REFERÊNCIAS}

AQUALAB. Analisador de atividade de água Decagon. Brasil: ABRASEQ, 1997. 21p. Manual, 01.

BENEDETTI, B.C.; JORGE, J.T. Curvas de umidade de equilíbrio de vários grãos. Ciência e Tecnologia de Alimentos, Campinas, v.7, n.2, p.172-188, 1987.

BRASIL. Ministério da Agricultura e Reforma Agrária. Regras para análise de sementes. Brasília: SNDA/DNDV/CLAV, 1992. 365p. 
BROOKER, D.B.; BAKKER-ARKEMA, F.W.; HALL, C.W. Grain equilibrium moisture content. In: Drying and storage of grains and oilseeds, New York, p.67-86, 1992.

CHINNAN, M.S.; BEAUCHAT, L.R. Sorption isotherms of whole cowpeas and flours. Lebensmittel-Wissenschaft und Technologie, London, v.18, p.83-8, 1985.

CHUNG, D.S.; PFOST, H.B. Adsorption and desorption of water vapor by cereal grains and their products. Part II. Development of the general isotherm equation. Transactions of the American Society Agricultural Engineers, v. 10, p.551-554, 1967.

HALL, C.W. Drying and storage of agricultural crops. Westport: AVI Publishing, 1980. 382p.

HALSEY, G. Physical adsorption on uniform surfaces. Journal of Chemical Physics, Woodbury, v.16, n.10, p.931-7, 1985.

LANGMUIR, I. Adsorption of gases on glass, mica and platinum. Journal of the American Chemical Society, n.46, p.1361-1403, 1918.

NELLIST, M.E.; HUGUES, M. Physical and biological processes in the drying of seed. Seed Science and Technology, Zürich, v. 1, n.3, p.613-643, 1973.

PARK, K.J.; NOGUEIRA, R.I. Modelos de ajuste de isotermas de sorção de alimentos. Engenharia Rural, Piracicaba, v.3, n.1, p.816, 1992.
PELEG, M. Assessment of a semi-empirical four parameter general model for sigmoid moisture sorption isotherms. Journal of Food Processing Engineering, Connecticut, v.16, n.1, p.21-37, 1993.

ROA, G.; ROSSI, S.J. Determinação experimental de curvas de teor de umidade de equilíbrio mediante a medição da umidade relativa de equilíbrio. Revista Brasileira de Armazenamento, Viçosa, v.2, n.2, p.17-22, 1977.

VALENTINI, S.R.T. Efeito da secagem de sementes de perobarosa (Apidosperma polyneuron M. Arg.). Campinas: 1992. $70 \mathrm{f}$. Dissertação (Mestrado em Engenharia Agrícola), Faculdade de Engenharia Agrícola, Universidade de Campinas, Campinas, 1992.

VAN DER BERG, C. Description of water activity of foods for engineering purposes by means of the GAB model of sorption. In: MCKENNA, B.M. (Ed.). Engineering and Food. London: Elsevier Applied Science, v.1, p.311-21, 1984.

VERTUCCI, C.W.; LEOPOLD, A.C. Water binding in legume seeds. Plant Physiology, Rockville, v.85, n.85, p. 224-231, 1987.

XIAORONG, H.; YUNLAN, Z.; CHENGLIAN, H.; MEI, T.; SHUPING, C. A comparison of methods for drying seeds: vacuum freeze-drier versus silica gel. Seed Science Research, v.8, n.1, p.29-33, 1998. Supplement. 